\title{
EXACT ARTIFICIAL BOUNDARY CONDITIONS FOR QUASILINEAR ELLIPTIC EQUATIONS IN UNBOUNDED DOMAINS*
}

\author{
HOUDE HAN ${ }^{\dagger}$, ZHONGYI HUANG ${ }^{\ddagger}$, AND DONGSHENG YIN $\$$
}

\begin{abstract}
To study the numerical solutions of quasilinear elliptic equations on unbounded domains in two or three dimensional cases, we introduce a circular or spherical artificial boundary. Based on the Kirchhoff transformation and the Fourier series expansion, the exact artificial boundary condition and a series of its approximations of the given quasilinear elliptic problem are presented. Then the original problem is equivalently or approximately reduced to a bounded computational domain. The well-posedness of the reduced problems are proved and the convergence results of our numerical solutions on bounded computational domain are given.
\end{abstract}

Key words. quasilinear elliptic equation, unbounded domain, artificial boundary condition

AMS subject classifications. $65 \mathrm{~N} 30,35 \mathrm{~J} 65$

\section{Introduction} problem

In this paper, we consider the numerical solution of the exterior quasilinear elliptic

$$
\left\{\begin{aligned}
-\nabla \cdot(a(x, u) \nabla u) & =f \quad \text { in } \Omega^{c} \equiv \mathbb{R}^{d} \backslash \Omega \\
\left.u\right|_{\partial \Omega} & =0
\end{aligned}\right.
$$

where $\Omega \subset \mathbb{R}^{d}(d=2$ or 3 ) is a bounded domain and $a(\cdot, \cdot)$ and $f$ are functions with various properties which will be specified later. Certainly, the problem (1.1) is not well posed. We need an additional boundary condition at infinity:

$$
\left\{\begin{array}{l}
u(x) \text { is bounded, as }|x| \rightarrow+\infty \quad(\text { for } d=2) \\
u(x) \rightarrow 0, \text { as }|x| \rightarrow+\infty \quad(\text { for } d=3)
\end{array}\right.
$$

Problem (1.1)-(1.2) has many physical applications in, for example, the field of heat transfer, where $a$ is the thermal conductivity of the medium and $u$ is the temperature field; the field of magnetostatics, where $a$ is the magnetic permeability and $u$ is the magnetic scalar potential; and the field of compressible flow, where $a$ is the density and $u$ is the velocity potential. There are many numerical results about problems of this kind with bounded domains $[2,4,12,13]$.

Suppose that the given function $a(\cdot, \cdot)$ satisfies $(c f .[10,12])$ :

$$
0<C_{0} \leq a(x, u) \leq C \quad \forall u \in \mathbb{R} \text {, and for almost all } x \in \Omega^{c}
$$

with two constants $C_{0}, C \in \mathbb{R}$, and

$$
|a(x, u)-a(x, v)| \leq C_{L}|u-v| \quad \forall u, v \in \mathbb{R} \text { and for almost all } x \in \Omega^{c},
$$

${ }^{*}$ Received: August 1, 2007; accepted (in revised version): October 28, 2007. Communicated by Shi Jin.

This work was partially supported by the NSFC Projects No. 10301017, 10471073 and 10676017 , the National Basic Research Program of China under the grant 2005CB321701, China Postdoctoral Science Foundation No. 20060390076.

$\dagger$ Dept. of Math., Tsinghua University, Beijing 100084, China (hhan@math.tsinghua.edu.cn).

¥Dept. of Math., Tsinghua University, Beijing 100084, China (zhuang@math.tsinghua.edu.cn).

$\S$ Dept. of Math., Tsinghua University, Beijing 100084, China (dyin@math.tsinghua.edu.cn). 
with a constant $C_{L}>0$. In the following, we suppose that the function $f \in L^{2}\left(\Omega^{c}\right)$ has compact support, i.e., there is a constant $R_{0}>0$, such that

$$
\operatorname{supp} f \subset \Omega_{R_{0}}=\left\{x \in \mathbb{R}^{d}|| x \mid \leq R_{0}\right\} .
$$

Furthermore, we assume that

$$
a(x, u) \equiv a_{0}(u) \quad \text { when }|x| \geq R_{0} .
$$

We introduce an artificial boundary in $\mathbb{R}^{d}$ :

$$
\Gamma_{R}=\left\{x \in \mathbb{R}^{d}|| x \mid=R\right\},
$$

with $R \geq R_{0} . \Gamma_{R}$ divides $\Omega^{c}$ into two parts, the bounded part $\Omega_{i}=\left\{x \in \Omega^{c}|| x \mid<R\right\}$ and the unbounded part $\Omega_{e}=\{x|| x \mid>R\}$. Then the problem (1.1)-(1.2) can be rewritten in the coupled form:

$$
\begin{aligned}
& \left\{\begin{aligned}
-\nabla \cdot(a(x, u) \nabla u) & =f \quad \text { in } \Omega_{i} \subset \mathbb{R}^{d}, \\
\left.u\right|_{\partial \Omega} & =0,
\end{aligned}\right. \\
& \left\{\begin{aligned}
-\nabla \cdot\left(a_{0}(u) \nabla u\right) & =0 \quad \text { in } \Omega_{e} \subset \mathbb{R}^{d}, \\
|u(x)| & \text { is bounded as }|x| \rightarrow+\infty \quad(\text { for } d=2), \\
|u(x)| & \rightarrow 0 \text { as }|x| \rightarrow+\infty \quad(\text { for } d=3) .
\end{aligned}\right.
\end{aligned}
$$

Moreover

$$
u(x) \text { and } a_{0}(u) \frac{\partial u}{\partial n} \text { are continuous on the artificial boundary } \Gamma_{R} .
$$

The problem (1.8)-(1.9) is a coupled problem, neither (1.8) nor (1.9) can be solved independently without the connecting condition (1.10). In the case that $a(x, u) \equiv a$ is independent of $x$ and $u$ when $|x| \geq R_{0}>0$, Wu, Kang and Yu have obtained the artificial boundary conditions on the circle $\left\{x \in \mathbb{R}^{2}|| x \mid=R \geq R_{0}\right\}$ [14]. In that case, the problem is simplified to the linear exterior elliptic problem. For additional related work, one can refer to the review paper [7] and the two books $[5,15]$. In this paper, we shall derive the artificial boundary conditions for more general quasilinear elliptic equations in two or three dimensional spaces.

First, we introduce the so-called Kirchhoff transformation [11]

$$
w(x)=\int_{0}^{u(x)} a_{0}(\xi) d \xi \quad \text { for } x \in \Omega_{e},
$$

then we get

$$
\nabla w=a_{0}(u) \nabla u .
$$

This means, from (1.9), that $w$ satisfies the following problem:

$$
\left\{\begin{array}{l}
-\Delta w=0 \quad \text { in } \Omega_{e} \subset \mathbb{R}^{d}, \\
|w(x)| \text { is bounded as }|x| \rightarrow+\infty, \quad(\text { for } d=2) \\
|w(x)| \rightarrow 0 \text { as }|x| \rightarrow+\infty . \quad(\text { for } d=3)
\end{array}\right.
$$

The remainder of this paper is organized as follows: In Section 2, we give the exact quasilinear artificial boundary conditions for two and three dimensional cases. In Section 3, we give the equivalent variational problems and the finite element approximations. The well-posedness and the convergence results of the reduced problems are proved. Finally, in Section 4, we present some numerical examples to show the efficiency and feasibility of our method. 


\section{Exact quasilinear artificial boundary conditions}

In this section, we shall derive the exact quasilinear artificial boundary conditions from the well-known exact artificial boundary conditions for Laplace's Equation [5, $7,9,15]$.

2.1. Exact quasilinear artificial boundary condition in $\mathbb{R}^{2}$. Suppose that $w(x)$ is the solution of the problem (1.13). If the value $\left.w\right|_{|x|=R}$ is given, namely

$$
\left.w\right|_{|x|=R}=w(R, \theta),
$$

then the problem (1.13) and (2.1) is well posed. For the solution in polar coordinates, $w(r, \theta)$, of the problem (1.13) and (2.1), we have the Fourier expansion [5, 9, 15]:

$$
w(r, \theta)=\frac{c_{0}}{2}+\sum_{n=1}^{\infty}\left(\frac{R}{r}\right)^{n}\left(c_{n} \cos n \theta+d_{n} \sin n \theta\right),
$$

with

$$
\begin{aligned}
& c_{n}=\frac{1}{\pi} \int_{0}^{2 \pi} w(R, \theta) \cos n \theta d \theta, n=0,1, \ldots \\
& d_{n}=\frac{1}{\pi} \int_{0}^{2 \pi} w(R, \theta) \sin n \theta d \theta, n=1,2, \ldots
\end{aligned}
$$

It is easy to get

$$
\left.\frac{\partial w}{\partial r}(r, \theta)\right|_{r=R}=-\frac{1}{R \pi} \sum_{n=1}^{\infty} n \int_{0}^{2 \pi} w(R, \varphi) \cos n(\varphi-\theta) d \varphi .
$$

From (1.12), we obtain

$$
\frac{\partial w}{\partial r}=a_{0}(u) \frac{\partial u}{\partial r}
$$

Combining (2.5) and (2.6), we get the exact boundary condition of $u$ on $\Gamma_{R}$,

$$
\begin{aligned}
\left.\left(a_{0}(u) \frac{\partial u}{\partial r}(r, \theta)\right)\right|_{r=R} & =-\frac{1}{R \pi} \int_{0}^{2 \pi} \sum_{n=1}^{\infty}\left(\int_{0}^{u(R, \varphi)} a_{0}(y) d y\right) n \cos n(\varphi-\theta) d \varphi \\
& \equiv \mathcal{K}_{\infty}(u(R, \theta))
\end{aligned}
$$

Substituting (2.7) into (1.8), we have

$$
\left\{\begin{aligned}
-\nabla \cdot(a(x, u) \nabla u) & =f, \quad \text { in } \Omega_{i} \subset \mathbb{R}^{2}, \\
\left.u\right|_{\partial \Omega} & =0, \\
\left.a_{0}(u) \frac{\partial u}{\partial n}\right|_{\Gamma_{R}} & =\mathcal{K}_{\infty}(u) .
\end{aligned}\right.
$$

Therefore, the solution of problem (2.8) is the restriction of the solution of problem (1.1) and (1.2) on the bounded domain $\Omega_{i}$. 
2.2. Exact quasilinear artificial boundary condition in $\mathbb{R}^{3}$. For the problem (1.13) with $d=3$, if the value $\left.w\right|_{|x|=R}$ is given in the spherical coordinates, namely

$$
\left.w\right|_{|x|=R}=w(R, \theta, \varphi),
$$

where

$$
\begin{array}{ll}
x_{1}=r \sin \theta \cos \varphi, & 0 \leq \theta \leq \pi, \\
x_{2}=r \sin \theta \sin \varphi, & 0 \leq \varphi \leq 2 \pi, \\
x_{3}=r \cos \theta, &
\end{array}
$$

then the solution of the problem (1.13) and (2.9), $w(r, \theta, \varphi)$, has the following expansion $[5,8,15]$ :

$$
\begin{aligned}
& w(r, \theta, \varphi) \\
= & \frac{c_{00}}{2} \frac{R}{r}+\sum_{n=1}^{\infty}\left(\frac{R}{r}\right)^{n+1}\left[\frac{c_{n 0}}{2} P_{n}^{0}(\cos \theta)+\sum_{m=1}^{n} P_{n}^{m}(\cos \theta)\left(c_{n m} \cos m \varphi+d_{n m} \sin m \varphi\right)\right]
\end{aligned}
$$

with

$$
\begin{aligned}
& c_{n m}=\frac{(2 n+1)(n-m) !}{2 \pi(n+m) !} \int_{0}^{2 \pi} \int_{0}^{\pi} w(r, \xi, \eta) P_{n}^{m}(\cos \xi) \cos m \eta \sin \xi d \xi d \eta, \\
& d_{n m}=\frac{(2 n+1)(n-m) !}{2 \pi(n+m) !} \int_{0}^{2 \pi} \int_{0}^{\pi} w(r, \xi, \eta) P_{n}^{m}(\cos \xi) \sin m \eta \sin \xi d \xi d \eta .
\end{aligned}
$$

Here $P_{n}^{0}(t)=P_{n}(t)$ is the Legendre polynomial of degree $n$,

$$
P_{n}^{0}(t)=P_{n}(t)=\frac{1}{2^{n} n !} \frac{d^{n}}{d t^{n}}\left(t^{2}-1\right)^{n},
$$

and $P_{n}^{m}(t)$ is the Legendre function,

$$
P_{n}^{m}(t)=\left(1-t^{2}\right)^{\frac{m}{2}} \frac{d^{m}}{d t^{m}} P_{n}(t)
$$

Furthermore, we have

$$
\begin{aligned}
\left.\frac{\partial w}{\partial r}\right|_{\Gamma_{R}}= & -\frac{c_{00}}{2 R} \\
& -\sum_{n=1}^{\infty} \frac{n+1}{R}\left[\frac{c_{n 0}}{2} P_{n}^{0}(\cos \theta)+\sum_{m=1}^{n} P_{n}^{m}(\cos \theta)\left(c_{n m} \cos m \varphi+d_{n m} \sin m \varphi\right)\right] .
\end{aligned}
$$

Using (2.10) and (2.11) and the addition formula of Legendre functions [6], we get

$$
\left.\frac{\partial w}{\partial r}(r, \theta, \varphi)\right|_{\Gamma_{R}}=-\int_{0}^{2 \pi} \int_{0}^{\pi} \sum_{n=0}^{\infty} w(R, \xi, \eta) \frac{(n+1)(2 n+1)}{4 \pi R} P_{n}(\cos \gamma) \sin \xi d \xi d \eta
$$

where

$$
\cos \gamma=\cos \xi \cos \theta+\sin \xi \sin \theta \cos (\eta-\varphi) .
$$


From (2.6), we obtain the exact boundary condition of $u$ on $\Gamma_{R}$,

$$
\begin{aligned}
& \left.\left(a_{0}(u) \frac{\partial u}{\partial r}\right)\right|_{\Gamma_{R}} \\
= & -\frac{1}{4 \pi R} \int_{0}^{2 \pi} \int_{0}^{\pi} \sum_{n=0}^{\infty}\left(\int_{0}^{u(R, \xi, \eta)} a_{0}(y) d y\right)(n+1)(2 n+1) P_{n}(\cos \gamma) \sin \xi d \xi d \eta \equiv \mathcal{K}_{\infty}(u) .
\end{aligned}
$$

Substituting (2.17) into (1.8), we have

$$
\left\{\begin{aligned}
-\nabla \cdot(a(x, u) \nabla u) & =f \quad \text { in } \Omega_{i} \subset \mathbb{R}^{3}, \\
\left.u\right|_{\partial \Omega} & =0, \\
\left.a_{0}(u) \frac{\partial u}{\partial n}\right|_{\Gamma_{R}} & =\mathcal{K}_{\infty}(u) .
\end{aligned}\right.
$$

\section{Finite element approximation}

3.1. The equivalent variational problems. First, let us focus on the problem (2.8) in $\mathbb{R}^{2}$. We will use $W^{m, p}(\Omega)$ denoting the standard Sobolev spaces,

$\|\cdot\|_{m, p, \Omega}$ and $|\cdot|_{m, p, \Omega}$ denoting the corresponding norms and semi-norms. In particular, we denote $H^{m}(\Omega)=W^{m, 2}(\Omega),\|\cdot\|_{m, \Omega}=\|\cdot\|_{m, 2, \Omega}$ and $|\cdot|_{m, \Omega}=|\cdot|_{m, 2, \Omega}$.

Let us introduce the space

$$
V=\left\{v \in H^{1}\left(\Omega_{i}\right)|v|_{\partial \Omega}=0\right\}
$$

and the corresponding norms

$$
\|v\|_{0, \Omega_{i}}=\sqrt{\int_{\Omega_{i}}|v|^{2} d x}, \quad\|v\|_{1, \Omega_{i}}=\sqrt{\int_{\Omega_{i}}\left(|v|^{2}+|\nabla v|^{2}\right) d x}
$$

The boundary value problem (2.8) is equivalent to the following variational problem:

$$
\left\{\begin{array}{l}
\text { Find } u \in V, \text { such that } \\
A(u ; u, v)+B(u ; u, v)=F(v) \quad \forall v \in V,
\end{array}\right.
$$

with

$$
\begin{aligned}
A(w ; u, v) & =\int_{\Omega_{i}} a(x, w) \nabla u \cdot \nabla v d x \\
B(w ; u, v) & =\int_{0}^{2 \pi} \int_{0}^{2 \pi} a_{0}(w(R, \varphi)) \frac{\partial u}{\partial \varphi}(R, \varphi) \frac{\partial v}{\partial \theta}(R, \theta) \sum_{n=1}^{\infty} \frac{\cos n(\varphi-\theta)}{n \pi} d \varphi d \theta, \\
F(v) & =\int_{\Omega_{i}} f(x) v(x) d x .
\end{aligned}
$$

Lemma 3.1. There is a constant $C>0$, such that

$$
\forall u, v, w \in V, \quad|B(w ; u, v)| \leq C\|u\|_{1, \Omega_{i}}\|v\|_{1, \Omega_{i}} \text { and } B(v ; v, v) \geq 0 .
$$

Proof. Using the equivalent norm theorem in [1], we have, for $s \in \mathbb{R}$,

$$
v \in H^{s}\left(\Gamma_{R}\right) \Longleftrightarrow v=\frac{c_{0}}{2}+\sum_{n=1}^{+\infty}\left(c_{n} \cos n \theta+d_{n} \sin n \theta\right), \frac{c_{0}^{2}}{2}+\sum_{n=1}^{+\infty}\left(1+n^{2}\right)^{s}\left(c_{n}^{2}+d_{n}^{2}\right)<+\infty .
$$


For $u, v \in V$, assume that

$$
\begin{gathered}
u(R, \varphi)=\frac{\alpha_{0}}{2}+\sum_{n=1}^{\infty}\left(\alpha_{n} \cos n \varphi+\beta_{n} \sin n \varphi\right), \\
v(R, \theta)=\frac{c_{0}}{2}+\sum_{n=1}^{\infty}\left(c_{n} \cos n \theta+d_{n} \sin n \theta\right),
\end{gathered}
$$

Then we obtain

$$
\begin{aligned}
& \frac{\partial u}{\partial \varphi}(R, \varphi)=\sum_{n=1}^{\infty} n\left(-\alpha_{n} \sin n \varphi+\beta_{n} \cos n \varphi\right) \\
& \frac{\partial v}{\partial \theta}(R, \theta)=\sum_{n=1}^{\infty} n\left(-c_{n} \sin n \theta+d_{n} \cos n \theta\right) .
\end{aligned}
$$

Combining property (1.3), Cauchy's inequality and the trace theorem [1], we get

$$
\begin{aligned}
|B(w ; u, v)| & \leq C_{1}\left[\sum_{n=1}^{\infty} n\left(\alpha_{n}^{2}+\beta_{n}^{2}\right)\right]^{1 / 2}\left[\sum_{n=1}^{\infty} n\left(c_{n}^{2}+d_{n}^{2}\right)\right]^{1 / 2} \\
& \leq C_{1}\|u\|_{1 / 2, \Gamma_{R}}\|v\|_{1 / 2, \Gamma_{R}} \leq C_{2}\|u\|_{1, \Omega_{i}}\|v\|_{1, \Omega_{i}}, \quad \forall u, v, w \in V .
\end{aligned}
$$

On the other hand, for any given $v \in V$, let us consider the following auxiliary problem in $\Omega_{e}$ :

$$
\left\{\begin{aligned}
-\nabla \cdot\left(a_{0}(u) \nabla u\right) & =0 \quad \text { in } \Omega_{e} \\
\left.u\right|_{\Gamma_{R}} & =\left.v\right|_{\Gamma_{R}} \\
u(x) & \text { is bounded as }|x| \rightarrow+\infty
\end{aligned}\right.
$$

From the analysis in Section 2, we know that the solution $u$ of the above problem (3.6) satisfies

$$
\left.a_{0}(u) \frac{\partial u}{\partial r}\right|_{\Gamma_{R}}=\mathcal{K}_{\infty}(u) \equiv \mathcal{K}_{\infty}(v)
$$

If we multiply Equation (3.6) by $u$ and integrate over $\Omega_{e}$, we arrive at

$$
B(v ; v, v)=\int_{\Omega_{e}} a_{0}(u)|\nabla u|^{2} d x \geq 0
$$

In practice, we need to truncate the series in (2.7) for some nonnegative integer $N \in \mathbb{Z}$,

$$
\left.\left(a_{0}(u) \frac{\partial u}{\partial r}(r, \theta)\right)\right|_{r=R}=\mathcal{K}^{N}(u)
$$

where

$$
\mathcal{K}^{N}(u)=-\frac{1}{R \pi} \int_{0}^{2 \pi} \sum_{n=1}^{N}\left(\int_{0}^{u(R, \varphi)} a_{0}(y) d y\right) n \cos n(\varphi-\theta) d \varphi
$$


So we only use the summation of the first $N$ terms in (2.7). We now focus on the approximate problem:

$$
\left\{\begin{aligned}
-\nabla \cdot\left(a\left(x, u^{N}\right) \nabla u^{N}\right) & =f \quad \text { in } \Omega_{i} \subset \mathbb{R}^{2}, \\
\left.u^{N}\right|_{\partial \Omega} & =0, \\
\left.a_{0}\left(u^{N}\right) \frac{\partial u^{N}}{\partial n}\right|_{\Gamma_{R}} & =\mathcal{K}^{N}\left(u^{N}\right) .
\end{aligned}\right.
$$

Certainly, problem (3.9) is equivalent to the following variational problem:

$$
\left\{\begin{array}{l}
\text { Find } u^{N} \in V \text { such that } \\
A\left(u^{N} ; u^{N}, v\right)+B_{N}\left(u^{N} ; u^{N}, v\right)=F(v) \quad \forall v \in V,
\end{array}\right.
$$

with

$$
B_{N}(w ; u, v)=\sum_{n=1}^{N} \frac{1}{n \pi} \int_{0}^{2 \pi} \int_{0}^{2 \pi} a_{0}(w(R, \varphi)) \frac{\partial u}{\partial \varphi}(R, \varphi) \frac{\partial v}{\partial \theta}(R, \theta) \cos n(\varphi-\theta) d \varphi d \theta .
$$

Similar to Lemma 3.1, we have:

Lemma 3.2. $\exists C>0$, s.t. $\forall u, v, w \in V,\left|B_{N}(w ; u, v)\right| \leq C\|u\|_{1, \Omega_{i}}\|v\|_{1, \Omega_{i}}$.

3.2. Finite element approximation. Suppose $\mathcal{T}_{h}$ is a regular and quasiuniform triangulation on $\Omega_{i}$, s.t.

$$
\Omega_{i}=\bigcup_{K \in \mathcal{T}_{h}} K
$$

where $K$ is a (curved) triangle; $h$ denotes the maximum side of the triangles. Let

$$
V_{h}=\left\{v_{h} \in V|v|_{K} \text { is a linear polynomial } \forall K \in \mathcal{T}_{h}\right\} .
$$

We then consider the approximate problem of (3.10),

$$
\left\{\begin{array}{l}
\text { Find } u_{h}^{N} \in V_{h} \text { such that } \\
A\left(u_{h}^{N} ; u_{h}^{N}, v_{h}\right)+B_{N}\left(u_{h}^{N} ; u_{h}^{N}, v_{h}\right)=F\left(v_{h}\right) \quad \forall v_{h} \in V_{h} .
\end{array}\right.
$$

From the theory of existence and uniqueness in [10], we have

Lemma 3.3. Problems (3.2), (3.10) and (3.14) have unique solvability.

From now on, let $u, u^{N} \in H^{2}\left(\Omega_{i}\right)$, and $u_{h}^{N} \in V_{h}$ be the solutions of problems (3.2), (3.10), and (3.14), respectively. To get the convergence result, cf. [10], we will also assume that

$$
V_{h} \subset V \bigcap W^{1, q}\left(\Omega_{i}\right), \quad \text { and } q=d+\varepsilon \text { for some } \varepsilon \in(0,1) .
$$

Furthermore, we require that $\left\{V_{h}\right\}_{h \rightarrow 0}$ is a family of finite-dimensional subspaces of $V \cap C\left(\Omega_{i}\right)$, such that

$$
\begin{aligned}
& \forall v \in V \bigcap C\left(\Omega_{i}\right), \quad \exists\left\{v_{h}\right\}_{h \rightarrow 0}: v_{h} \in V_{h}, \quad\left\|v-v_{h}\right\|_{1, \Omega_{i}} \rightarrow 0, \text { as } h \rightarrow 0, \\
& \text { and }\left\|v_{h}\right\|_{1, q, \Omega_{i}} \leq C(v) \quad \forall h,
\end{aligned}
$$

where $C(v)>0$ is independent of $h$. 
REMARK 3.1. The continuous piecewise polynomial spaces, such as (3.13), satisfy the condition (3.15). And if we let $v_{h}=\Pi_{h} v$, where $\Pi_{h}: V \rightarrow V_{h}$ is the interpolation operator, we have

$$
\left\|v_{h}\right\|_{1, q, \Omega_{i}} \leq\left\|\Pi_{h} v-v\right\|_{1, q, \Omega_{i}}+\|v\|_{1, q, \Omega_{i}} \leq C(v)
$$

From the convergence theory in [10], it is easy to obtain the following convergence result:

$$
\forall N \geq 0, \quad \lim _{h \rightarrow 0}\left\|u_{h}^{N}-u^{N}\right\|_{1, \Omega_{i}}=0 \quad \text { and } \quad u^{N} \in V \bigcap W^{1, q}\left(\Omega_{i}\right) .
$$

Furthermore, we can get the following lemma:

LEMma 3.4. We have the following convergence result:

$$
\lim _{N \rightarrow \infty}\left\|u-u^{N}\right\|_{1, \Omega_{i}}=0 .
$$

Proof. First, from Proposition (1.3) and Lemma 3.1, we have

$$
\begin{aligned}
\left\|u^{N}\right\|_{1, \Omega_{i}}^{2} & \leq C\left[A\left(u^{N} ; u^{N}, u^{N}\right)+B\left(u^{N} ; u^{N}, u^{N}\right)\right] \\
& =C\left[F\left(u^{N}\right)+B\left(u^{N} ; u^{N}, u^{N}\right)-B_{N}\left(u^{N} ; u^{N}, u^{N}\right)\right] \\
& \leq C\left[\|f\|_{0, \Omega_{i}} \cdot\left\|u^{N}\right\|_{1, \Omega_{i}}+\left|B\left(u^{N} ; u^{N}, u^{N}\right)-B_{N}\left(u^{N} ; u^{N}, u^{N}\right)\right|\right] .
\end{aligned}
$$

For $u^{N} \in V$, assume that

$$
\begin{aligned}
& w^{N}(r, \varphi)=\int_{0}^{u^{N}(r, \varphi)} a_{0}(\xi) d \xi=\frac{\alpha_{0}}{2}+\sum_{n=1}^{\infty}\left(\frac{R_{0}}{r}\right)^{n}\left(\alpha_{n} \cos n \varphi+\beta_{n} \sin n \varphi\right) \quad \forall r \geq R_{0}, \\
& u^{N}(R, \theta)=\frac{c_{0}}{2}+\sum_{n=1}^{\infty}\left(c_{n} \cos n \theta+d_{n} \sin n \theta\right) .
\end{aligned}
$$

We obtain that

$$
\begin{aligned}
& \left|B\left(u^{N} ; u^{N}, u^{N}\right)-B_{N}\left(u^{N} ; u^{N}, u^{N}\right)\right| \\
= & \left|\int_{0}^{2 \pi} \int_{0}^{2 \pi} \frac{\partial w^{N}}{\partial \varphi}(R, \varphi) \frac{\partial u^{N}}{\partial \theta}(R, \theta) \sum_{n=N+1}^{\infty} \frac{\cos n(\varphi-\theta)}{n \pi} d \varphi d \theta\right| \\
= & \left|\sum_{n=N+1}^{\infty}\left(\frac{R_{0}}{R}\right)^{n} n \pi\left(\alpha_{n} c_{n}+\beta_{n} d_{n}\right)\right| \\
\leq & \pi\left(\frac{R_{0}}{R}\right)^{N+1}\left[\sum_{n=N+1}^{\infty} n\left(\alpha_{n}^{2}+\beta_{n}^{2}\right)\right]^{1 / 2}\left[\sum_{n=1}^{\infty} n\left(c_{n}^{2}+d_{n}^{2}\right)\right]^{1 / 2} \\
\leq & C_{1}\left(\frac{R_{0}}{R}\right)^{N+1}\left\|w^{N}\right\|_{\frac{1}{2}, \Gamma_{R_{0}}}\left\|u^{N}\right\|_{\frac{1}{2}, \Gamma_{R}} \\
\leq & C_{2}\left(\frac{R_{0}}{R}\right)^{N+1}\left\|u^{N}\right\|_{1, \Omega_{i}}^{2} .
\end{aligned}
$$


Because $R>R_{0}$, we know that $\left\{u^{N}\right\}_{N=0}^{\infty}$ is bounded in $V$. Therefore, we have a subsequence $\left\{u^{N_{n}}\right\}$ such that $u^{N_{n}} \rightarrow \bar{u} \in V$. Furthermore, $\forall v \in V \cap C^{\infty}\left(\Omega_{i}\right)$,

$$
\begin{aligned}
& |A(\bar{u} ; \bar{u}, v)+B(\bar{u} ; \bar{u}, v)-F(v)| \\
\leq & \left|A(\bar{u} ; \bar{u}, v)-A\left(\bar{u} ; u^{N_{n}}, v\right)\right|+\left|A\left(\bar{u} ; u^{N_{n}}, v\right)-A\left(u^{N_{n}} ; u^{N_{n}}, v\right)\right| \\
& +\left|B(\bar{u} ; \bar{u}, v)-B_{N_{n}}(\bar{u} ; \bar{u}, v)\right|+\left|B_{N_{n}}(\bar{u} ; \bar{u}, v)-B_{N_{n}}\left(\bar{u} ; u^{N_{n}}, v\right)\right| \\
& +\left|B_{N_{n}}\left(\bar{u} ; u^{N_{n}}, v\right)-B_{N_{n}}\left(u^{N_{n}} ; u^{N_{n}}, v\right)\right| \\
\leq & \left|A\left(\bar{u} ; \bar{u}-u^{N_{n}}, v\right)\right|+\left|B_{N_{n}}\left(\bar{u} ; \bar{u}-u^{N_{n}}, v\right)\right|+C\left[\left\|\bar{u}-u^{N_{n}}\right\|_{0, \Omega_{i}} \cdot\left\|u^{N_{n}}\right\|_{1, \Omega_{i}} \cdot\|v\|_{1, \infty, \Omega_{i}}\right. \\
& \left.+\left(\frac{R_{0}}{R}\right)^{N_{n}+1}\|\bar{u}\|_{1, \Omega_{i}} \cdot\|v\|_{1, \Omega_{i}}\right] \rightarrow 0, \quad \text { as } n \rightarrow \infty .
\end{aligned}
$$

Consequently, we get

$$
A(\bar{u} ; \bar{u}, v)+B(\bar{u} ; \bar{u}, v)=F(v) \quad \forall v \in V \bigcap C^{\infty}\left(\Omega_{i}\right) .
$$

As $V \cap C^{\infty}\left(\Omega_{i}\right)$ is a dense set of $V$, we know that $\bar{u}$ is a solution of problem (3.2). From the unique solvability of problem (3.2), we know that any weak cluster point of the sequence $\left\{u^{N}\right\}_{N=0}^{\infty}$ coincides with the solution. Consequently, $u^{N} \rightarrow u$. On the other hand, we have

$$
\begin{aligned}
& \left\|u-u^{N}\right\|_{1, \Omega_{i}}^{2} \leq C_{1} A\left(u, u-u^{N}, u-u^{N}\right) \\
= & C_{1}\left[A\left(u^{N}, u^{N}, u-u^{N}\right)+B_{N}\left(u^{N}, u^{N}, u-u^{N}\right)-B\left(u, u, u-u^{N}\right)-A\left(u, u^{N}, u-u^{N}\right)\right] \\
\leq & C_{2}\left[\left\|u-u^{N}\right\|_{0, p, \Omega_{i}} \cdot\left\|u^{N}\right\|_{1, q, \Omega_{i}} \cdot\left\|u-u^{N}\right\|_{1, \Omega_{i}}+\left\|u-u^{N}\right\|_{\frac{1}{2}, \Gamma_{R}} \cdot\left\|u-u^{N}\right\|_{1, \Omega_{i}}\right. \\
& \left.+\left(\frac{R_{0}}{R}\right)^{N+1}\|u\|_{1, \Omega_{i}} \cdot\left\|u-u^{N}\right\|_{1, \Omega_{i}}\right] .
\end{aligned}
$$

Using the compactness of the embedding operator $H^{1}\left(\Omega_{i}\right) \hookrightarrow L^{p}\left(\Omega_{i}\right)$ and of the trace operator $\gamma: W^{1, q}\left(\Omega_{i}\right) \rightarrow H^{\frac{1}{2}}\left(\Gamma_{R}\right)$, we arrive at

$$
\lim _{N \rightarrow \infty}\left\|u-u^{N}\right\|_{1, \Omega_{i}}=0 .
$$

Finally, we get the following convergence result:

THEOREM 3.1. Let $u \in H^{2}\left(\Omega_{i}\right)$, and let assumptions (3.15)-(3.17) be satisfied. Then we have:

$$
\lim _{h \rightarrow 0, N \rightarrow \infty}\left\|u-u_{h}^{N}\right\|_{1, \Omega_{i}}=0
$$

REMARK 3.2. For the three dimensional spaces case, we can get similar convergence results. 


\section{Numerical example}

In this section, we give some numerical examples to show the feasibility and efficiency of our method. We choose the linear finite element space as given in (3.13).

EXAMPLE 4.1. First, let us consider the problem

$$
\left\{\begin{aligned}
-\nabla \cdot(a(x, u) \nabla u) & =f(x) \quad \text { for } x \in \Omega^{c} \equiv \mathbb{R}^{2} \backslash \Omega, \\
\left.u\right|_{\partial \Omega} & =0,
\end{aligned}\right.
$$

where $\Omega=\left\{x=(r, \theta) \in \mathbb{R}^{2} \mid r \leq 1\right\}$, and for $x=(r, \theta) \in \Omega^{c}$,

$$
\begin{aligned}
a(x, u) & = \begin{cases}1+r^{2}+\frac{1}{1+u^{2}}, & 1 \leq r \leq 2 ; \\
5+\frac{1}{1+u^{2}}, & r>2\end{cases} \\
f(x) & = \begin{cases}4-r^{2}, & 1 \leq r \leq 2 \\
0, & r>2\end{cases}
\end{aligned}
$$

It is easy to check that $a(\cdot, \cdot)$ and $f$ satisfy the conditions (1.3)-(1.6). Here we let $R=3$, i.e., we introduce an artificial boundary $\Gamma_{R}=\left\{x \in \mathbb{R}^{2}|| x \mid=3\right\}$, and we solve the problem on the domain $\Omega_{i}=\left\{x \in \mathbb{R}^{2}|1 \leq| x \mid \leq 3\right\}$. Furthermore, we let $\Delta r=\frac{2}{M}$, $\Delta \theta=\frac{2 \pi}{3 M}$ for some integer $M \in \mathbb{N}$. In this case, because $u(r, \theta)$ is independent of $\theta$, we can only use $N=0$ to get the optimal approximation results, $c f$. Table 4.1. The exact solution ' $u$ ' is solved with $N=100$ and a very fine mesh: $\Delta r=\frac{1}{64}, \Delta \theta=\frac{\pi}{192}$.

TABLE 4.1. Spatial discretization error test at $N=0$ for example 4.1.

\begin{tabular}{c|cccc}
\hline mesh size $\triangle r$ & $1 / 4$ & $1 / 8$ & $1 / 16$ & $1 / 32$ \\
\hline$\left\|u_{h}^{N}-u\right\|_{H^{1}\left(\Omega_{i}\right)}$ & $2.34 \mathrm{E}-1$ & $1.18 \mathrm{E}-1$ & $6.01 \mathrm{E}-2$ & $3.03 \mathrm{E}-2$ \\
\hline convergence order & & 1.0 & 1.0 & 1.0 \\
\hline$\left\|u_{h}^{N}-u\right\|_{L^{2}\left(\Omega_{i}\right)}$ & $1.85 \mathrm{E}-1$ & $5.03 \mathrm{E}-2$ & $1.230 \mathrm{E}-2$ & $2.36 \mathrm{E}-3$ \\
\hline convergence order & & 1.9 & 2.0 & 2.4 \\
\hline
\end{tabular}

EXAMPLE 4.2. Then, let us consider the problem

$$
\left\{\begin{aligned}
-\nabla \cdot(a(x, u) \nabla u) & =f(x) \quad \text { for } x \in \Omega^{c} \equiv \mathbb{R}^{2} \backslash \Omega, \\
\left.u\right|_{\partial \Omega} & =0,
\end{aligned}\right.
$$

where $\Omega=\left\{x=(r, \theta) \in \mathbb{R}^{2} \mid r \leq 1\right\}$, and for $x=(r, \theta) \in \Omega^{c}$,

$$
\begin{aligned}
a(x, u) & = \begin{cases}1+(2-r) \sin ^{2} \theta+\exp \left(-u^{2}\right), & 1 \leq r \leq 2 ; \\
1+\exp \left(-u^{2}\right), & r>2 ;\end{cases} \\
f(x) & = \begin{cases}(2-r)|\theta-\pi|^{2}, & 1 \leq r \leq 2 ; \\
0, & r>2 .\end{cases}
\end{aligned}
$$

It is also easy to check that $a(\cdot, \cdot)$ and $f$ satisfy the conditions (1.3)-(1.6). Here we also set $R=3$, i.e., we solve the problem on the domain $\Omega_{i}=\left\{x \in \mathbb{R}^{2}|1 \leq| x \mid \leq 3\right\}$. Furthermore, we let $\Delta r=\frac{2}{M}, \Delta \theta=\frac{2 \pi}{3 M}$ for some integer $M \in \mathbb{N}$. In this case, we find that we can use $N=10$ to get the almost optimal convergence rate, $c f$. Figure 4.1. The exact solution ' $u$ ' is solved with $N=100$ and very fine mesh: $\Delta r=\frac{1}{64}, \Delta \theta=\frac{\pi}{192}$. 


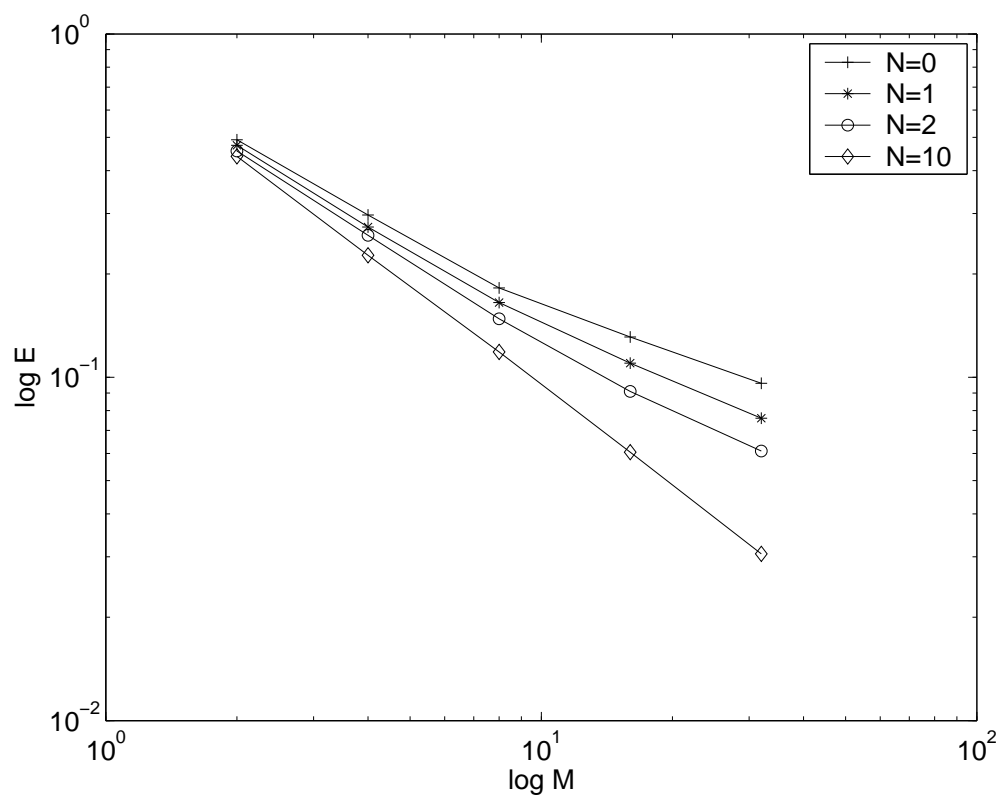

FIG. 4.1. The convergence rate of the artificial boundary with different truncation terms for example 4.2. Here $E=\left\|u_{h}^{N}-u\right\|_{H^{1}\left(\Omega_{i}\right)}$.

EXAMPLE 4.3. Finally, let us consider the problem

$$
\left\{\begin{aligned}
-\nabla \cdot(a(x, u) \nabla u) & =f(x), \quad \text { for } x \in \Omega^{c} \equiv \mathbb{R}^{2} \backslash \Omega \\
\left.u\right|_{\partial \Omega} & =g(x),
\end{aligned}\right.
$$

where $\Omega=\left\{x=\left(x_{1}, x_{2}\right) \in \mathbb{R}^{2} \mid x_{1}^{2}+x_{2}^{2} \leq 1\right\}$, and for $x=\left(x_{1}, x_{2}\right)$,

$$
\begin{aligned}
& a(x, u)= \begin{cases}4-|x|^{2}+\frac{1}{1+u^{2}}, & 1 \leq|x| \leq 2 ; \\
\frac{1}{1+u^{2}}, & |x|>2 ;\end{cases} \\
& f(x)= \begin{cases}2 x_{2}|x|^{2} \frac{4-|x|^{2}\left(1+|x|^{2}\right)-x_{2}^{2}}{\left(|x|^{4}+x_{2}^{2}\right)^{2}}, & 1 \leq|x| \leq 2 ; \\
0, & |x|>2 ;\end{cases} \\
& g(x)=\tan \left(x_{2}\right), \quad \text { for } x \in \partial \Omega \text {. }
\end{aligned}
$$

The exact solution of (4.3) is

$$
u(x)=\tan \left(\frac{x_{2}}{|x|^{2}}\right) .
$$

It easy to see that $a(\cdot, \cdot)$ does not satisfy the condition (1.3). But we can see that our numerical results are also very much satisfied, $c f$. Figure 4.2-4.3 and Table 4.2. In our numerics, we set $R=2$, i.e., we introduce an artificial boundary $\Gamma_{R}=\left\{x \in \mathbb{R}^{2}|| x \mid=2\right\}$, and we solve the problem on the domain $\Omega_{i}=\left\{x \in \mathbb{R}^{2}|1 \leq| x \mid \leq 2\right\}$. Furthermore, we let $\Delta r=\frac{1}{M}, \Delta \theta=\frac{2 \pi}{6 M}$ for some integer $M \in \mathbb{N}$. The numerical results are given in Table 4.2 and Figures 4.2-4.3.

REMARK 4.1. If we use the higher order finite element spaces and the solution has higher regularities, we can get a higher order convergence rate. 
82 ARTIFICIAL BOUNDARY CONDITIONS FOR QUASILINEAR ELLIPTIC EQUATIONS

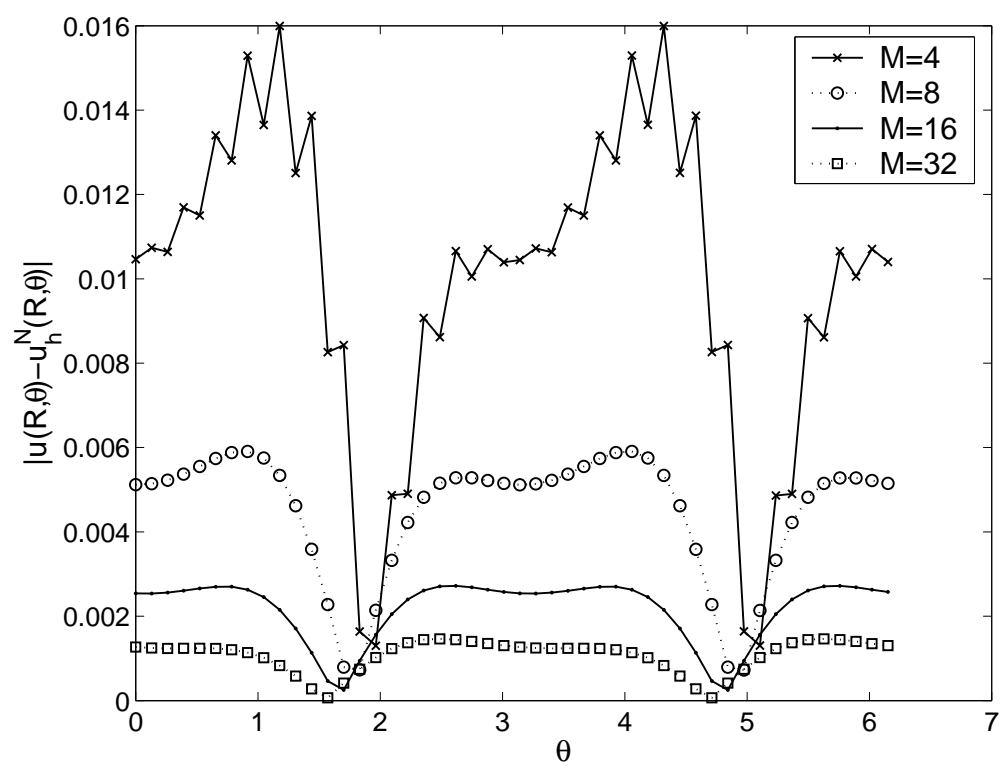

FIG. 4.2. Example 4.3: the errors on the artificial boundary with different mesh sizes. Here we let $N=10, \Delta r=\frac{1}{M}, \Delta \theta=\frac{2 \pi}{6 M}$.

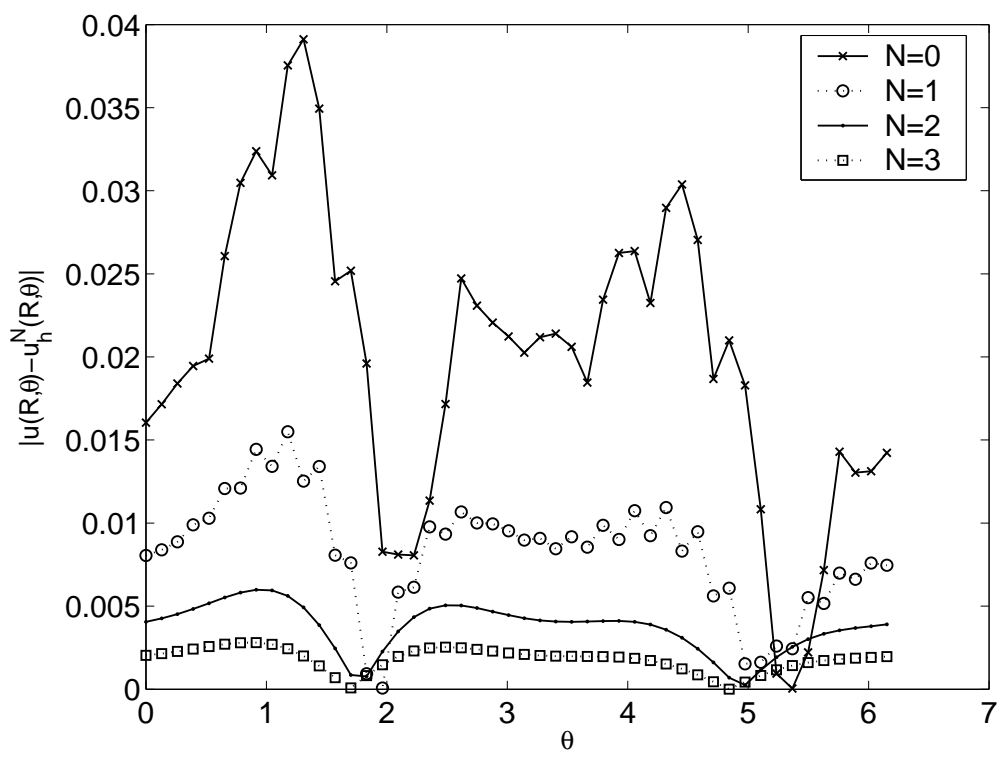

Fig. 4.3. Example 4.3: the errors on the artificial boundary with different $N$. Here we let $M=32, \Delta r=\frac{1}{M}, \Delta \theta=\frac{2 \pi}{6 M}$.

\section{Conclusion}

In this paper, we propose the exact artificial boundary conditions of a generic quasilinear elliptic equation on unbounded domains in $\mathbb{R}^{2}$ (or $\mathbb{R}^{3}$ ). We assume that 
TABLE 4.2. Spatial discretization error test at $N=10$ for example 4.3.

\begin{tabular}{|c|c|c|c|c|}
\hline mesh size $\triangle r$ & $1 / 4$ & $1 / 8$ & $1 / 16$ & $1 / 32$ \\
\hline$\left\|u_{h}^{N}-u\right\|_{H^{1}\left(\Omega_{i}\right)}$ & $1.71 \mathrm{E}-1$ & $8.48 \mathrm{E}-2$ & $3.81 \mathrm{E}-2$ & $1.82 \mathrm{E}-2$ \\
\hline convergence order & & 1.0 & 1.2 & 1.1 \\
\hline$\left\|u_{h}^{N}-u\right\|_{L^{2}\left(\Omega_{i}\right)}$ & $6.61 \mathrm{E}-2$ & $1.68 \mathrm{E}-2$ & $4.19 \mathrm{E}-3$ & $1.03 \mathrm{E}-3$ \\
\hline convergence order & & 2.0 & 2.0 & 2.0 \\
\hline
\end{tabular}

the source term has compact support. After we introduce a circular or spherical artificial boundary, we get a boundary problem on a disc or a ball enclosed by the artificial boundary which is exactly the restriction of the original problem on this bounded domain. Based on the Kirchhoff transformation, Fourier series expansion and the special functions techniques, we obtain the exact artificial boundary conditions and a series of approximating artificial boundary conditions. We also prove the wellposedness and the convergence results of the reduced problems with the artificial boundary conditions. Our numerical examples show the efficiency of our method.

\section{REFERENCES}

[1] R.A. Adams, Sobolev Spaces, New York: Academic Press, 1975.

[2] S.S. Chow, Finite element error estimates for non-linear elliptic equations of monotone type, Numer. Math., 54, 373-393, 1989.

[3] P.G. Ciarlet, The Finite Element Method for Elliptic Problems, Amsterdam: North-Holland publishing company, 1978.

[4] M. Feistauerl and A. Ženíšek, Finite element solution of nonlinear elliptic problems, Numer. Math., 50, 451-475, 1987.

[5] D. Givoli, Numerical Methods for Problems in Infinite Domains, Elsevier, Amsterdam, 1992.

[6] I.S. Gradshteyn and I.M. Ryzhik, Table of Integrals, Series, and Products, Academic Press, San Diego, 2000.

[7] H. Han, The artifical boundary method - numerical solutions of partial differential equations on unbounded domains, in Frontiers and Prospects of Contemporary Applied Mathematics edited by T. Li and P. Zhang, Higher Education Press, World Scientific, 33-58, 2005.

[8] H. Han, C. He and X. Wu, Analysis of artificial boundary conditions for exterior boundary value problems in three dimensions, Numer. Math., 85, 367-386, 2000.

[9] H. Han and X. Wu, Approximation of infinite boundary conditions and its application to finite element mathods, J. Comput. Math., 3, 179-192, 1985.

[10] I. Hlaváček, M. Kř́ížek and J. Malý, On Galerkin approximations of a quasi-linear nonpotential elliptic problem of a nonmonotone type, J. Math. Anal. Appl., 184, 168-189, 1994.

[11] D.B. Ingham and M.A. Kelmanson, Boundary Integral Equation Analyses of Singular, Potential, and Biharmonic Problems, C.A. Brebbia and S.A. Orszag eds., Lecture Notes in Engineering, 7, Springer-Verlag Beilin, Heidelberg, 1984.

[12] S. Korotov and M. Křížek, Finite element analysis of variational crimes for a quasilinear elliptic problem in 3D, Numer. Math., 84, 549-576, 2000.

[13] W. Liu, Finite element approximation of a nonlinear elliptic equation arising from bimaterial problems in elastic-plastic mechanics, Numer. Math., 86, 491-506, 2000.

[14] Z.P. Wu, T. Kang and D.H. Yu, On the coupled NBEM and FEM for a class of nonlinear exterior Dirichlet problem in $\mathbb{R}^{2}$, Science in China, Series A, Mathematics, 47, 181-189, 2004.

[15] D.H. Yu, Natural Boundary Integral Method and its Applications, Science Press/Kluwer Academic Publishers, 2002. 